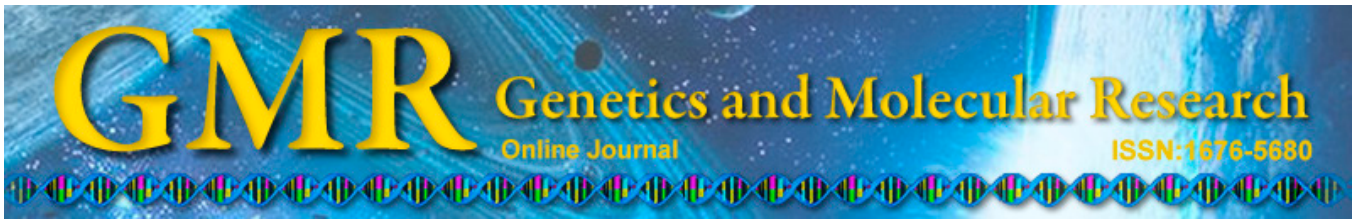

\title{
Elucidating the function and tolerance mechanism of gamma delta $(\gamma \delta)$ T cells in a Helicobacter pylori infection model
}

\author{
T.E. Chen ${ }^{1}$, X.M. Xu' ${ }^{2}$, P. Liu ${ }^{1}$, S.Y. Liang ${ }^{2}$ and W.X. Lv ${ }^{1}$ \\ ${ }^{1}$ Critical Care Medical Center, Ningbo No. 2 Hospital, Ningbo, China \\ ${ }^{2}$ Department of Microbiology, Ningbo No. 2 Hospital, Ningbo, China \\ Corresponding author: X.M. Xu \\ E-mail: XiaominMedicine@163.com
}

Genet. Mol. Res. 14 (3): 10543-10552 (2015)

Received February 5, 2015

Accepted May 28, 2015

Published September 8, 2015

DOI http://dx.doi.org/10.4238/2015.September.8.16

\begin{abstract}
In this study, the functions and mechanisms of $\gamma \delta$ T cells were analyzed in patients infected with Helicobacter pylori. Peripheral blood was collected from gastritis patients in the Gastroenterology Department of Ningbo No. 2 Hospital. Preliminary analyses revealed $24 \mathrm{H}$. pylori-positive and $17 \mathrm{H}$. pylori-negative patients. The wild-type and $\gamma \delta \mathrm{T}$ knockout mice were infected with cultured $H$. pylori cells (obtained from the H. pylori-positive patients). H. pylori in mice was quantified by polymerase chain reaction; gastritis was confirmed by hematoxylin and eosin staining. The TCR- $\delta^{--r}$ mice were treated with vein adoptive immunotherapy $24 \mathrm{~h}$ prior to $H$. pylori inoculation; the same method was used to detect the extent of gastritis and bacterial colonization. The $\gamma \delta$ T knockout mice showed high levels of $H$. pylori infection than the wild-type mice; in addition, the knockout mice showed severe disease pathology. $\gamma \delta \mathrm{T}$ knockout mice also displayed increased matrix metalloproteinase-9 (MMP-9) and decreased MMP-7 expression in the gastric mucosa. $\gamma \delta \mathrm{T}$ cells play a protective role in patients infected with H. pylori. $\gamma \delta$ T cell [responsible for the production of interleukin-17 (IL-17) and IL-22] expression was increased in $H$.
\end{abstract}


pylori-positive patients, indicating statistical significance. However, there was no significant difference in interferon-gamma $+\gamma \delta \mathrm{T}$ expression between the positive and negative patients. This study demonstrated the probable involvement of $\gamma \delta$ T cells in the immune response of an organism, via the secretion of IL-17 and IL-22.

Key words: $\gamma \delta$ T cell receptor; Helicobacter pylori infection; Immune response

\section{INTRODUCTION}

T cell receptors (TCR) are molecules found on the surface of $\mathrm{T}$ cells that are responsible for antigen recognition and mediate immune response. Based on the protein chains comprising the TCRs T cells are classified into the alpha beta $(\alpha \beta)$ and gamma delta $(\gamma \delta)$ T cell categories (Cho et al., 2010; Chung et al., 2010). $\gamma \delta$ T cells are rarely observed in the peripheral blood, spleen, and lymph nodes (Cornelissen et al., 2009). These cells mainly exist in the subcutaneous tissues, the urinary and reproductive tract, skin, digestive and respiratory mucous membranes, and the lymph tissues. Although the absolute number of $\gamma \delta$ T cells is lesser than that of $\alpha \beta$ $\mathrm{T}$ cells, previous studies have reported that the immune response of $\gamma \delta \mathrm{T}$ cells is more rapid (Davey et al., 2011; Dejima et al., 2011; D'Elios et al., 2014); in fact, this is a key function of the $\gamma \delta$ T cells. Therefore, $\gamma \delta$ T cells mainly engage in the immune defense and block the invasion of pathogens (DeLyria et al., 2009; Do et al., 2012; Dubin et al., 2012). Pathogenic invasion of the human body results in the activation of $\gamma \delta \mathrm{T}$ cells, which leads to the rapid multiplication of $\gamma \delta \mathrm{T}$ cells (in a short period of time). These cells then target the site of pathogenic infection. Helicobacter pylori is a Gram-negative, microaerophilic, spiral-shaped bacterium, which mainly causes inflammation of gastric mucosa. This study investigated the effect of $\gamma \delta$ T cells against H. pylori infection (Eberl et al., 2009); we also conducted a preliminary investigation into the immune regulatory mechanisms against the $H$. pylori colonization in infected individuals.

\section{MATERIAL AND METHODS}

\section{Sample collection}

Peripheral blood samples were collected from gastritis patients at the Gastroenterology clinic of the Ningbo No. 2 Hospital between June 2012 and May 2013. Preliminary analyses identified $24 \mathrm{H}$. pylori-positive and $17 \mathrm{H}$. pylori-negative cases. The patients were selected based on the absence of ulcers, tuberculosis, cancer, or other illnesses (including viral infections); in addition, the patients were not treated with antibiotics for at least 1 month prior to the sample collection. This study was approved by the Ethics Committee of the Department of the Ningbo No. 2 Hospital. Signed informed consent forms were obtained from all patients prior to sample collection and experimentation.

\section{Mice and reagents}

H. pylori colonies were extracted from the gastric mucosa of infected patients. The third-generation adaptive colonies were inoculated into wild-type mice. Specific pathogen-free 
experimental mice (C57BL/6 background), weighing between 16 and $22 \mathrm{~g}$ and aged between 6 and 8 weeks, were obtained from the Laboratory Animal Center of our hospital. All culture media, serum, and reagents used in this study were purchased from Hyclone Laboratories, Inc. (South Logan, UT, USA).

\section{Mouse groups}

Six mice were included in the $\gamma \delta$ T knockout group; these mice weighed between 16 and $22 \mathrm{~g}$ and were between 6 and 8 weeks old.The wild-type mouse group consisted of 6 mice, weighing 16-21 g and aged between 6 and 8 weeks. There were no statistically significant differences between the two groups.

\section{Experimental procedures}

\section{Establishment of $\mathrm{H}$. pylori infection in the mouse model}

The bacterial samples were obtained and cultivated in liquid culture media (Hopebio, HB8647). Subsequently, cultured bacterial cells were used to vaccinate the mice via gastric filling.

\section{Mouse tissue samples and cell separation}

H. pylori-infected mice were killed at different time points; the spleen lymphocytes and lymph node cells were extracted from the dead mice.

\section{Quantitative polymerase chain reaction (qPCR)}

One microgram of total RNA was extracted and then reverse-transcribed with iScript Reverse Transcription Supermix (Bio-Rad). cDNA products were mixed with iQ SYBR green Supermix (Bio-Rad). The qPCR was performed under the following reaction conditions: initial denaturation at $90^{\circ} \mathrm{C}$ for $30 \mathrm{~s}$, and 40 cycles of denaturation at $95^{\circ} \mathrm{C}$ for $5 \mathrm{~s}$, annealing at $60^{\circ} \mathrm{C}$ for 5 $\mathrm{s}$, and extension at $72^{\circ} \mathrm{C}$ for $30 \mathrm{~s}$. The primers used for gene amplification are listed in Tables 1 and 2 .



\section{Flow cytometric analysis of phenotype and intrinsic factors of $T$ cells}

The isolated lymphocytes were stimulated and centrifuged for separation. Antibodies including anti-CD3 antibody (Abcam, ab16669), anti-interferon gamma (IFN- $\gamma$ ) antibody 
(Abcam, ab87030), anti-IL-17 antibody (Abcam, ab118869), anti-IL-22 RA2 antibody (Abcam, ab203211) against specific cell surface molecules were added to the collected lymphocytes, in order to stain the cell surface. After being fixed and washed twice, the cells were stained with cell factor antibodies (subsequently washed twice). The stained samples were tested via flow cytometry (BD FACSAria ${ }^{\mathrm{TM}}$ Fusion).

\begin{tabular}{lc} 
Table 2. Real-time polymerase chain reaction system. \\
\hline cDNA & $4 \mu \mathrm{L}$ \\
\hline 2X Real-time PCR Master Mix & 10 \\
Upstream primer $(5 \mu \mathrm{M})$ & 1 \\
Downstream primer $(5 \mu \mathrm{M})$ & 1 \\
ddH $_{2} \mathrm{O}$ & 4 \\
Total volume & 20 \\
\hline
\end{tabular}

\section{Magnetic bead separation}

Fc-receptor blockers were added in the suspended lymphocytes and incubated for $15 \mathrm{~min}$ at room temperature. The appropriate fluorescein isothiocyanate-labeled antibodies (Abcam) and beads were added to these lymphocytes. After totally being mixed, the samples were incubated for $10 \mathrm{~min}$ at room temperature, and then for the magnetic bead separation.

\section{Determining $\gamma \delta \mathrm{T}$ cell function in $H$. pylori-infected mice}

\section{Development of $\gamma \delta$ T cell knockout mice}

The mice were killed by cervical dislocation 28 days after infection with $H$. pylori colonies. The killed mice were soaked in 75\% ethanol for 1-3 min and their abdomens were opened. The mice were subjected to gastric separation surgery (Romrell et al., 1975); a piece of the gastric mucosa was removed from the greater curvature of the stomach, and the samples fixed with 19\% methanol. The remaining tissue samples were divided into 3 equal parts,wrapped carefully with foil, and stored in liquid nitrogen (for backup). DNA was extracted from the gastric tissues using the QIAmp DNA MiniKit (Tiangen Biotech Co., Ltd., Beijing, China), in accordance with the manufacturer instructions. The H. pylori colonization in the gastric tissues of mice was analyzed by PCR, as detailed in a previous study (Eberl et al., 2009). Briefly, the appropriate probes and primers were synthesized, the 16S DNA standard samples were prepared, and the standard samples of PCR and 16S DNA expression were quantified using the probe method. The $\mathrm{C}_{\mathrm{T}}$ and $\log$ values obtained for the standard samples were used to plot the standard curve equation, and the number of bacteria in gastric tissues was quantitated.

\section{$\gamma \delta$ T cell adaptive immunotherapy}

The adaptive immunotherapy strategies were tested in mice by injecting each mouse with approximately $2.5 \times 10^{5} \gamma \delta \mathrm{T}$ cells (retinal venous injection). The mice were infected again with $H$. pylori after $24 \mathrm{~h}$. 


\section{Analysis of $\gamma \delta \mathrm{T}$ cell expression in $H$. pylori-infected patients}

Blood samples were collected from all H. pylori-infected patients; these were subjected to anticoagulant treatment. The treated blood was subjected to high speed centrifugation. The collected blood cells were diluted with Hank's balanced salt solution (HBSS) (Gibco), and centrifuged again at $400 \mathrm{~g}$ for $20 \mathrm{~min}$. The intermediate white coating (platelets, lymphocytes, monocytes, and granulocytes) was carefully obtained and washed twice with HBSS. The cell concentration was quantitated for flow cytometry analysis.

\section{Statistical analysis}

All data were processed using the SPSS statistical software platform (IBM, Armonk, NY, USA). The data were analyzed by analysis of variance (ANOVA) and the $\chi^{2}$ test. P values $<0.05$ were considered to be statistically significant.

\section{RESULTS}

\section{Lack of $\gamma \delta$ T cells negatively impacts host response to $H$. pylori infection}

We observed a significant increase in bacterial colonization in the knockout mice 28 days after $H$. pylori inoculation; in addition, the knockout mice displayed inflammatory reaction of greater severity compared to the wild-type mice; this difference was observed to be statistically significant $(P<0.05)$. Careful observation of the gastric tissues revealed the obvious infiltration of inflammatory cells in the sub-mucosa of the knockout mice, compared to that seen in the wild-type mice. In addition, the knockout mice showed a (statistically) significant increase in disease pathology. The results suggested that $\gamma \delta$ T cells may play a major protective role in patients/mice infected with $H$. pylori (Figure 1).
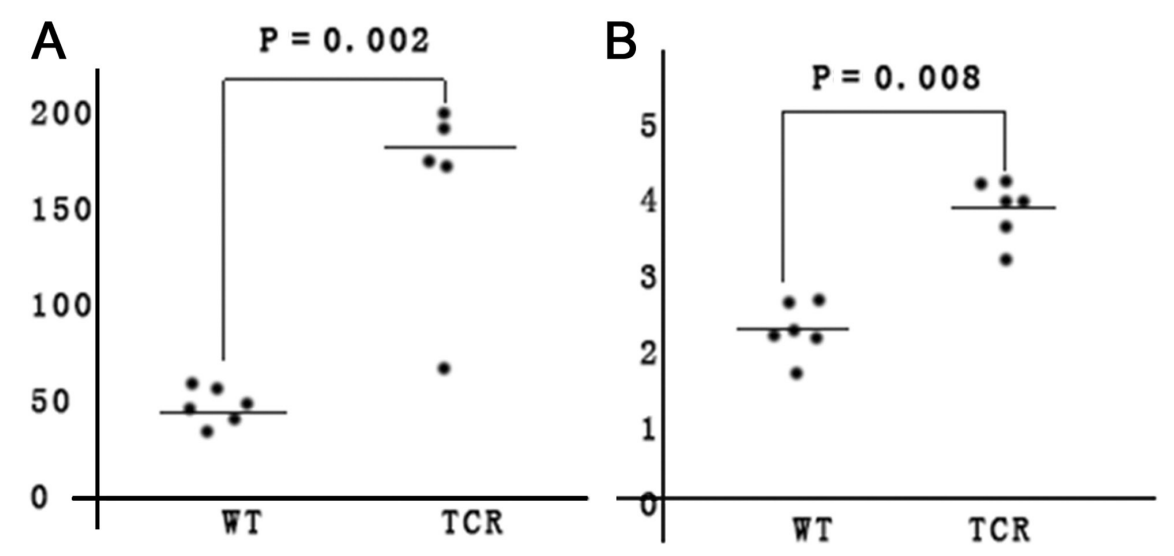

Figure 1. Effects of $\gamma \delta \mathrm{T}$ cell knockout in the colonization of Helicobacter pylori and inflammation of gastric tissue. A. Count of the colonized H. pylori in mouse stomach; B. histological score of gastritis (28 days postbacterial inoculation). $\mathrm{WT}=$ wild type $\mathrm{TCR}=\mathrm{T}$ cell receptor. 


\section{$\gamma \delta \mathrm{T}$ cells (secreted in response to $H$. pylori infection) influence local MMP expression}

We observed an increase in the expression of MMP-9, and a decrease in MMP-7 expression, in the stomach of knockout mice $(\mathrm{P}<0.05)$. This difference was statistically significant. No other statistically significant difference was observed in the expression of relevant indicators $(\mathrm{P}>0.05$; Figure 2$)$.

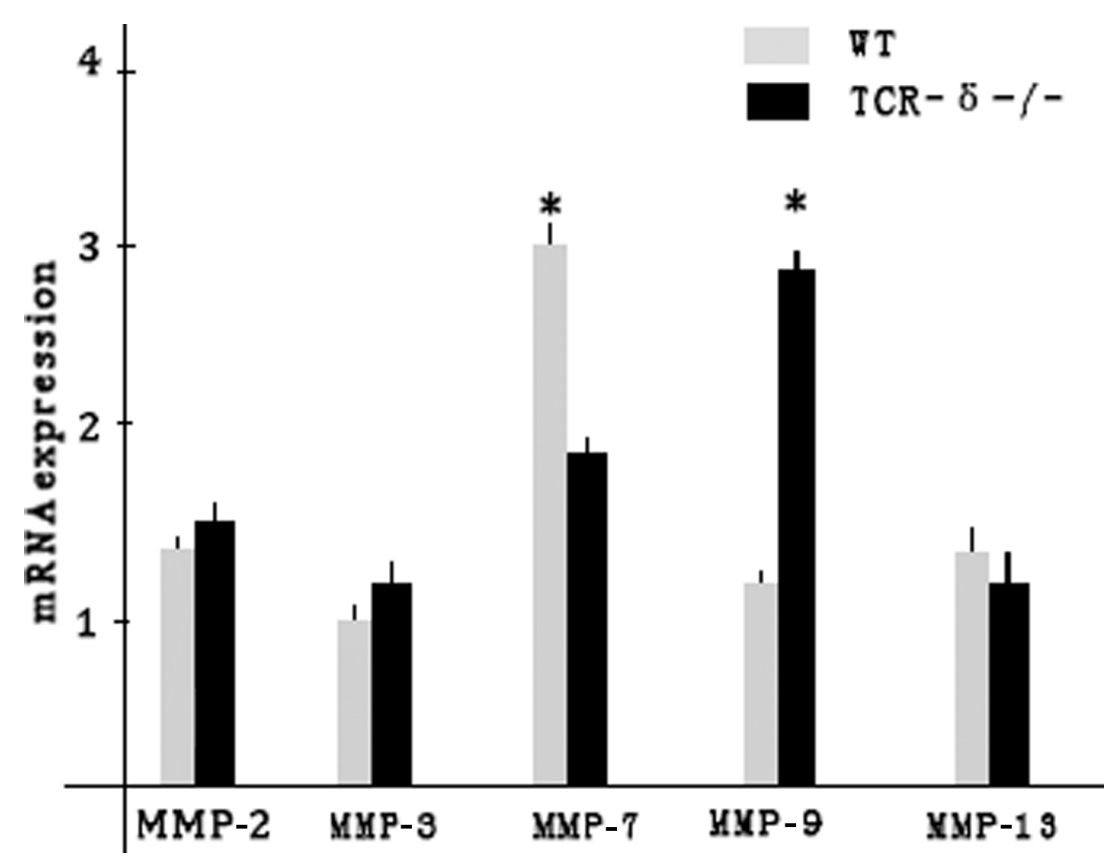

Figure 2. Matrix metalloproteinase (MMP) expression in the gastric mucosa of Helicobacter pylori-infected mice. $\mathrm{WT}=$ wild type; $\mathrm{TCR}=\mathrm{T}$ cell receptor.

\section{Flow cytometric detection of $\gamma \delta \mathrm{T}$ cell ratio in peripheral blood}

No obvious (statistically significant) changes were detected in the $\gamma \delta \mathrm{T}$ cell content in the peripheral blood of patients infected with $H$. pylori, compared to those in the uninfected patients $(\mathrm{P}=0.7886)$.

\section{Effect of $H$. pylori infection on cytokine secretion by $\gamma \delta \mathrm{T}$ cells}

A few of the $\gamma \delta \mathrm{T}$ cells detected in the human peripheral blood of $H$. pylori-positive patients co-expressed IL-17, IL-22, and IFN- $\gamma$, compared to the cells observed in the blood obtained from $H$. pylori-negative patients. Statistical analyses revealed a significant increase in the ratio of IL-17+, IL-22+, and IL-17+ IL-22+- $\gamma \delta$ T cells in the peripheral blood of $H$. pylori-positive patients, compared to that in the blood samples of $H$. pylori-negative patients. This indicated that $\gamma \delta$ T cells might be involved in the immune response to H. pylori infection through the secretion of IL-17 and IL-22 (Figure 3 and Table 3). 

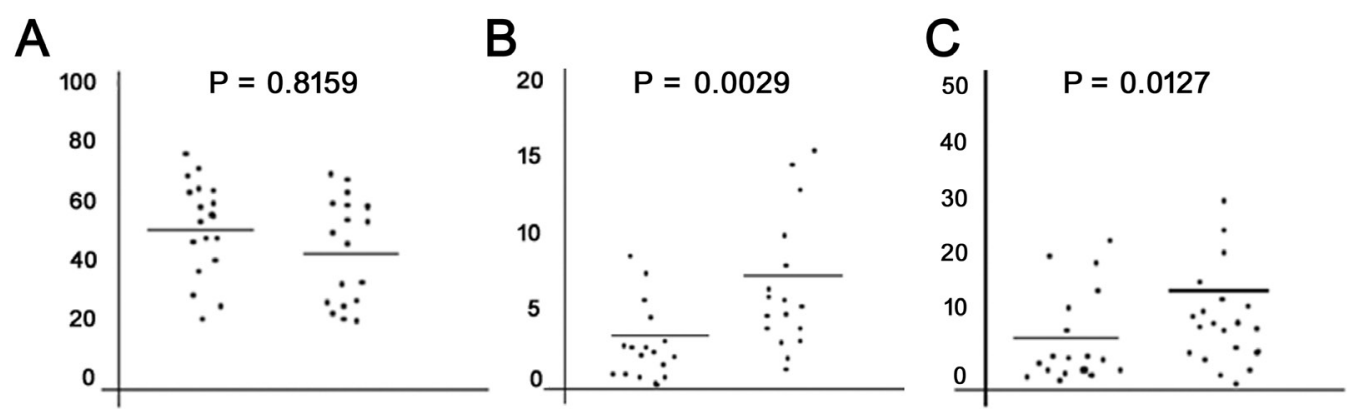

Figure 3. Detection of A. interferon gamma (IFN- $\gamma)+-$; B. interleukin 17 (IL-17) +-; and C. IL-22+- cytokine secreted by $\gamma \delta$ T cells.

Table 3. Proportion of various cellular and molecular isoforms of $\gamma \delta$ T cells.
\begin{tabular}{lccccccc} 
Group & Number & $\begin{array}{c}\text { IFN- } \gamma \\
(\%)\end{array}$ & $\begin{array}{c}\text { IL-17 } \\
(\%)\end{array}$ & $\begin{array}{c}\text { IL-22 } \\
(\%)\end{array}$ & $\begin{array}{c}\text { IFN- } \gamma \text { and } \\
\text { IL-22 }(\%)\end{array}$ & $\begin{array}{c}\text { IFN- } \gamma \text { and } \\
\text { IL-17 }(\%)\end{array}$ & $\begin{array}{c}\text { IF-17 and } \\
\text { IL-22 }(\%)\end{array}$ \\
\hline Helicobacter pylori-positive patients & 24 & 46.30 & 6.13 & 11.30 & 4.20 & 2.90 & 3.60 \\
H. pylori-negative patients & 17 & 43.50 & 2.70 & 6.40 & 1.40 & 1.20 & 1.50 \\
$\chi^{2}$ & & 2.64 & 4.25 & 5.18 & 1.87 & 3.76 & 6.45 \\
P value & & 0.63 & 0.034 & 0.018 & 0.079 & 0.14 & 0.027 \\
\hline
\end{tabular}

IFN- $\gamma=$ interferon gamma; IL-17 = interleukin 17; IL-22 = interleukin 22.

\section{Ratio of the cell cytokine content secreted by $\gamma \delta \mathrm{T}$ cells and $\mathrm{T}$ cells}

$\gamma \delta \mathrm{T}$ cell proportions in the peripheral blood of patient and control samples were analyzed from CD3+ lymphocytes by making gates of IFN- $\gamma+$, IL-17+ and IL-22+. The results indicated that the $\gamma \delta \mathrm{T}$ cell subset was a very small portion of the whole T lymphocytes in peripheral blood. The ratios of IFN- $\gamma+$ or IL-22+ T lymphocytes in peripheral blood the $H$. pylori-positive and -negative patients showed no significant difference, whereas there was a significant increase in the ratio of IL-17+ $\gamma \delta$ T cells in $H$. pylori-negative patients. These results indicated that the IL-17+ $\gamma \delta \mathrm{T}$ cells participated in $H$. pylori immune response, despite presenting a very small ratio of the total $\mathrm{T}$ lymphocyte count (Table 4 and Figure 4).

Table 4. Proportions of cytokines secreted by $\gamma \delta$ T cells.
\begin{tabular}{lcccc}
\hline Group & Number & IFN- $\gamma(\%)$ & IL-17 (\%) & IL-22 (\%) \\
\hline Helicobacter pylori-positive patients & 24 & 10.80 & 14.70 & 3.2 \\
H. pylori-negative patients & 17 & 9.50 & 7.90 & 2.1 \\
$\chi^{2}$ & & 2.50 & 4.80 & 3.4 \\
P value & & 0.07 & 0.03 & 0.1 \\
\hline
\end{tabular}

IFN- $\gamma=$ interferon gamma; IL-17 = interleukin 17; IL-22 = interleukin 22.

\section{Secretion of IL-17 by $\gamma \delta$ T cells was dependent on IL-23}

There were no obvious changes in the IL-17+ $\gamma \delta$ T cells and IFN- $\gamma+\gamma \delta$ T cell content in the spleen of uninfected mice $48 \mathrm{~h}$ after inoculation; during the same time period, there was no 
obvious decrease in the IL-17+ $\gamma \delta$ T cell content in knockout mice. The difference of IL-17+ $\gamma \delta$ $\mathrm{T}$ cell counts between the knockout and wild-type mice was statistically significant. There were no obvious changes in the proportions of IL-17+ and $\gamma \delta$ T cells. We observed a statistically significant decrease in the expression of ROR $\gamma \mathrm{T}$ cells in the knockout mice. We observed an increase in the expression of IL-23 in wild-type mice; however, IL-23 was not expressed in the knockout mice, which indicated its role in the secretion of IL-17+ $\gamma \delta$ T cells (Table 4 and Figure 5).

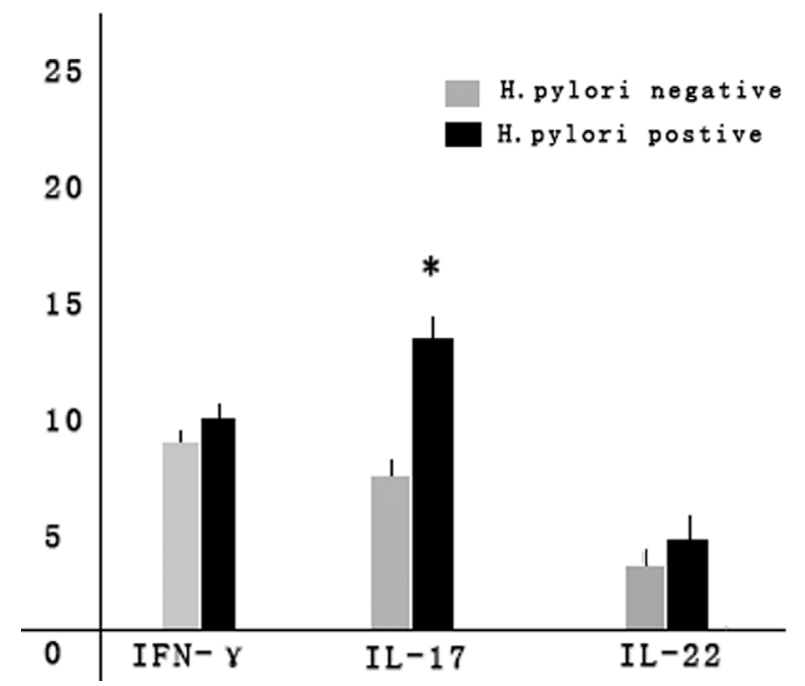

Figure 4. Proportions of $\gamma \delta$ T cells secreting interferon gamma (IFN- $\gamma$ ), interleukin (IL)-17, and IL-22. The figure exhibits the ratios of $\gamma \delta$ T cells secreting different cytokines to the total T cells in the Helicobacter pylori-positive and -negative groups; $* \mathrm{P}<0.05$.
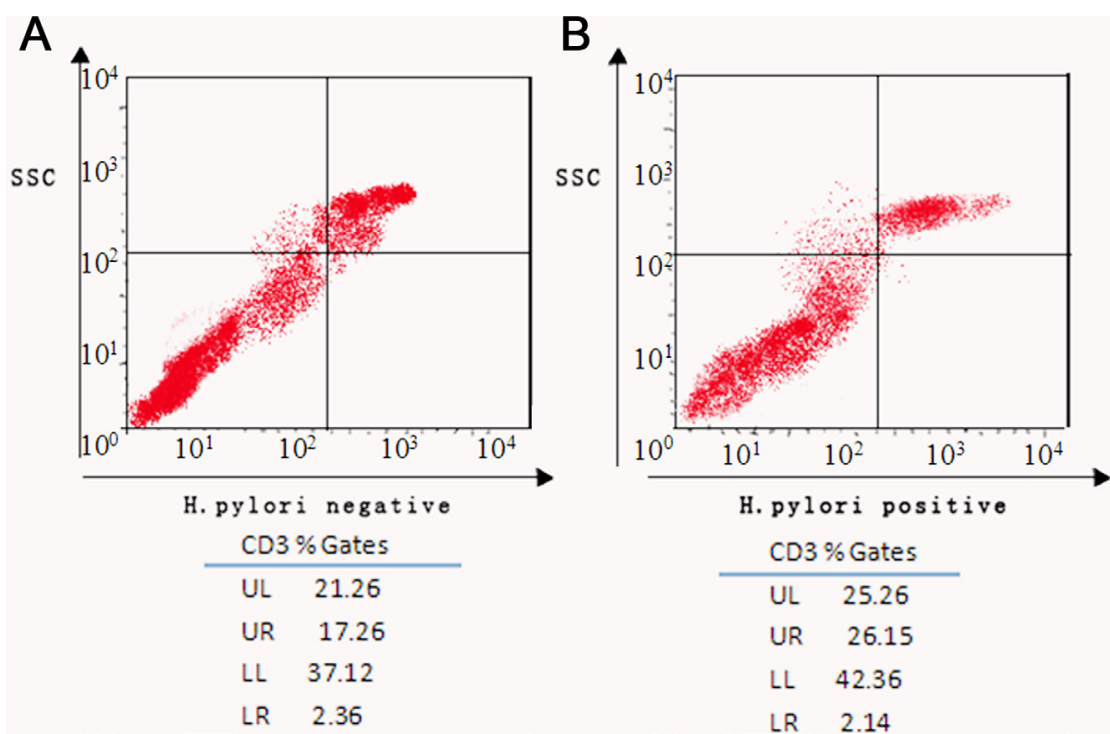

Figure 5. Flow cytometric analysis on $\gamma \delta \mathrm{T}$ cells (using the CD3+ T cell population as the lymphocyte gate) in Helicobacter pylori-negative patients (A) and H. pylori-positive patients (B).. 


\section{DISCUSSION}

Inflammation, caused by pathogenic infection, resulted in the secretion of a large number of lymphocytes to the area of infection, in order to check the spread of pathogens. Previous studies (Fenoglio et al., 2009; Fang et al., 2010; Fujiwara et al., 2012) have illustrated the involvement of $\gamma \delta$ T cells in early-stage immune mechanisms; in case of a localized infection, there was a rapid increase in $\gamma \delta$ T cell expression during the early stages of infection. In this study, we attempted to illustrate the effect of $\gamma \delta$ T cells on $H$. pylori colonization, and resulting inflammation, in gastric tissues. A thorough analysis of the experimental results indicated that $\gamma \delta \mathrm{T}$ cells might function as barriers in the immune response (Huber, 2010; Inagaki-Ohara et al., 2011; Kuroda et al., 2012). However, we could not identify the specific functions of the different subtypes; therefore, these must be elucidated in future research attempts. Some previous studies have demonstrated the role of $\gamma \delta$ T cells in the pathological impairment of Chlamydia trachomatis; these results can be combined with those obtained in this study. Both studies suggested that the $\gamma \delta$ T cells might serve as dual functions, and attempt to identify the regulatory, strength and time of immune response, and the subtypes of $\gamma \delta$ T cells produced. Pathogen types and period sought to be considered to elucidate the functions of $\gamma \delta \mathrm{T}$ cells in bacterial infection as well as each phase of the disease. Previous studies analyzing the peripheral blood of $H$. pylori-positive and -negative patients reported no fluctuations in the $\gamma$ $\delta \mathrm{T}$ cell proportion between the two; however, other studies (Li et al., 2012; Ma et al., 2012) reported a 2.4-fold increase in the $\gamma \delta \mathrm{T}$ cell content in $H$. pylori-positive patients than in the $H$. pylori-negative patients, which was inconsistent with the results of our study. This difference could be attributed to the lack of samples or the different stages of $H$. pylori infection (Romi et al., 2011).The $H$. pylori infection periods can be broadly classified into the transient infection and sustained colonization periods. $\gamma \delta$ T cells are mainly produced during these two periods; however, $\gamma \delta$ T cells play different roles during the two periods. Based on this theory, different H. pylori infection periods were believed to determine the timing and strength of the $\gamma \delta \mathrm{T}$ cell response. The results of this study indicated a statistically significant decrease in the ratio of IL-17+ $\gamma \delta$ T cells in knockout mice $48 \mathrm{~h}$ after infection, compared to the wild-type mice. We also observed a statistically significant decrease in the expression of ROR $\gamma$ T cells in knockout mice. We also observed an increase in the IL-23 expression in wild-type mice; on the other hand, IL-23 was not expressed in knockout mice, which indicated that IL-23 largely functioned in IL-17+ $\gamma \delta \mathrm{T}$ cells. This also suggested that the $\gamma \delta \mathrm{T}$ cell immune response to H. pylori infection was in conjunction with the secretion of IL-17 and IL-22. However, the mechanism of cytokine secretion and the effect of cytokines on $\mathrm{T}$ cell function remain to be elucidated. A greater volume of peripheral blood is required to conduct more in-depth analyses of $\gamma \delta$ T cell expression and function during bacterial infection.

In summary, we analyzed the $\gamma \delta$ T cell function during H. pylori infection, and deduced that $\mathrm{T}$ cells play a protective role during bacterial infections. These cells are mainly involved in cytokine-mediated immune response to the infecting pathogen. This study primarily analyzed the H. pylori infection; further research could focus on infection by other types of bacteria, in order to expand the available knowledge regarding $\gamma \delta \mathrm{T}$ cell immune response.

\section{Conflicts of interest}

The authors declare no conflict of interest. 


\section{ACKNOWLEDGMENTS}

Research supported by the Project of Medical Science in Ningbo (\#2013A14) and the Project of Social Development in Ningbo (\#2011C51002).

\section{REFERENCES}

Cho JS, Pietras EM, Garcia NC, Ramos RI, et al. (2010). IL-17 is essential for host defense against cutaneous Staphylococcus aureus infection in mice. J. Clin. Invest. 120: 1762-1773.

Chung WC, Jung SH, Lee KM, Paik CN, et al. (2010). The detection of Helicobacter pylori cag pathogenicity islands (PAIs) and expression of matrix metalloproteinase-7 (MMP-7) in gastric epithelial dysplasia and intramucosal cancer. Gastric Cancer 13: 162-169.

Cornelissen F, Mus AM, Asmawidjaja PS, van Hamburg JP, et al. (2009). Interleukin-23 is critical for full-blown expression of a non-autoimmune destructive arthritis and regulates interleukin-17A and RORgammat in gammadelta T cells. Arthritis Res. Ther. 11: R194.

D’Elios MM and Czinn SJ (2014). Inflammation, immunity, and vaccines for Helicobacter pylori. Helicobacter 19: 21-28.

Davey MS, Lin CY, Roberts CW, Heuston S, et al. (2011). Human neutrophil clearance of bacterial pathogens triggers anti-microbial gammadelta T cell responses in early infection. PLoS Pathog. 7: e1002040.

Dejima T, Shibata K, Yamada H, Hara H, et al. (2011). Protective role of naturally occurring interleukin-17A-producing $\gamma \delta \mathrm{T}$ cells in the lung at the early stage of systemic candidiasis in mice. Infect. Immun. 79: 4503-4510.

DeLyria ES, Redline RW and Blanchard TG (2009). Vaccination of mice against $H$. pylori induces a strong Th-17 response and immunity that is neutrophil dependent. Gastroenterology 136: 247-256.

Do JS, Visperas A, O’Brien RL and Min B (2012). CD4 T cells play important roles in maintaining IL-17-producing $\gamma \delta$ T-cell subsets in naive animals. Immunol. Cell Biol. 90: 396-403.

Dubin PJ, Martz A, Eisenstatt JR, Fox MD, et al. (2012). Interleukin-23-mediated inflammation in Pseudomonas aeruginosa pulmonary infection. Infect. Immun. 80: 398-409.

Eberl M and Moser B (2009). Monocytes and gammadelta T cells: close encounters in microbial infection. Trends Immunol. 30: 562-568.

Eberl M, Roberts GW, Meuter S, Williams JD, et al. (2009). A rapid crosstalk of human gammadelta T cells and monocytes drives the acute inflammation in bacterial infections. PLoS Pathog. 5: e1000308.

Fang H, Welte T, Zheng X, Chang GJ, et al. (2010). Gammadelta T cells promote the maturation of dendritic cells during West Nile virus infection. FEMS Immunol. Med. Microbiol. 59: 71-80.

Fenoglio D, Poggi A, Catellani S, Battaglia F, et al. (2009). Vdelta1 T lymphocytes producing IFN-gamma and IL-17 are expanded in HIV-1-infected patients and respond to Candida albicans. Blood 113: 6611-6618.

Fujiwara S, Nagai H, Oniki S, Yoshimoto T, et al. (2012). Interleukin (IL)-17 versus IL-27: opposite effects on tumor necrosis factor- $\alpha$-mediated chemokine production in human keratinocytes. Exp. Dermatol. 21: 70-72.

Huber SA (2010). $\gamma \delta$ T lymphocytes kill T regulatory cells through CD1d. Immunology 131: 202-209.

Inagaki-Ohara K, Sakamoto Y, Dohi T and Smith AL (2011). $\gamma \delta \mathrm{T}$ cells play a protective role during infection with Nippostrongylus brasiliensis by promoting goblet cell function in the small intestine. Immunology 134: 448-458.

Kuroda H, Saito H and Ikeguchi M (2012). Decreased number and reduced NKG2D expression of V $\delta 1 \gamma \delta \mathrm{T}$ cells are involved in the impaired function of V $\delta 1 \gamma \delta \mathrm{T}$ cells in the tissue of gastric cancer. Gastric Cancer 15: 433-439.

Li Z, Zhang C, Zhou Z, Zhang J, et al. (2012). Small intestinal intraepithelial lymphocytes expressing CD8 and T cell receptor $\gamma \delta$ are involved in bacterial clearance during Salmonella enterica serovar Typhimurium infection. Infect. Immun. 80: 565-574.

Ma Y, Aymeric L, Locher C, Mattarollo SR, et al. (2012). Contribution of IL-17-producing gamma delta T cells to the efficacy of anticancer chemotherapy. J. Exp. Med. 80: 491-503.

Romi B, Soldaini E, Pancotto L, Castellino F, et al. (2011). Helicobacter pylori induces activation of human peripheral $\gamma \delta+$ T lymphocytes. PloS One 6: e19324.

Romrell LJ, Coppe MR, Munro DR and Ito S (1975). Isolation and separation of highly enriched fractions of viable mouse gastric parietal cells by velocity sedimentation. J. Cell Biol. 65: 428-438. 